\title{
Electromyographic analysis according to shoe weight during ambulation
}

\author{
Eunsang Lee ${ }^{a}$, Byunghoon Lee ${ }^{b}$, Juchul Cho ${ }^{a, c}$, Yongwoo Lee $^{d}$, Seungwon Lee ${ }^{d}$ \\ ${ }^{a}$ Department of Physical Therapy, The Graduate School, Sahmyook University, Seoul, Republic of Korea \\ ${ }^{b}$ Department of Physical Therapy, Gwangju Health University, Gwangju, Republic of Korea \\ ${ }^{\mathrm{C}}$ Rehabilitation Team, Konyang University Hospital, Daegu, Republic of Korea \\ ${ }^{\mathrm{d} D e p a r t m e n t}$ of Physical Therapy, Sahmyook University, Seoul, Republic of Korea
}

Objective: To examine the effects of different shoe weights on lower leg muscle fatigue when walking by electromyographic (EMG) analysis due to the most effective weight for loading not being established.

Design: Cross-sectional study.

Methods: Thirty healthy university students (15 male, 15 female) were enrolled and randomly assigned into three conditions, which included wearing athletic shoes with an addition of $300 \mathrm{~g}, 500 \mathrm{~g}$, and 1,000 g weights respectively. Prior to walking, all subjects were instructed to sit in a chair for 10 minutes. All subjects walked at a speed of $3.6 \mathrm{~m} / \mathrm{s}$ on a treadmill for 20 minutes without rest. EMG measurements were taken using the median power frequency to assess for the effect of the different weight of shoes on muscle fatigue of the soleus, gastrocnemius, and tibialis anterior while walking on a treadmill in an upright posture. EMG measurements were taken during the first and last 30 seconds of walking.

Results: In terms of muscle fatigue, for the soleus, the median power frequency was significantly lower with $1,000 \mathrm{~g}$ compared with $300 \mathrm{~g}$ and $500 \mathrm{~g}(p<0.05)$. For the tibialis anterior, the median power frequency was significantly lower with $1,000 \mathrm{~g}$ than 300 $\mathrm{g}$ and $500 \mathrm{~g}(p<0.05)$. For the gastrocnemius, the median power frequency was significantly lower with $1,000 \mathrm{~g}$ compared with 300 $\mathrm{g}(p<0.05)$.

Conclusions: Increased shoe weight increases soleus, gastrocnemius, and tibialis anterior muscle fatigue during ambulation.

Key Words: Electromyography, Gait, Lower extremity, Shoe

\section{Introduction}

Increased energy intake and reduced physical activity are responsible for numerous chronic diseases, such as obesity, diabetes, ischemic heart disease and so on, worldwide [1]. Accordingly, several governments and non-governmental organizations worldwide have emphasized the importance of increasing physical activity, and have provided guidelines for physical activity as an essential aspect to improving health. These guidelines also emphasize the importance of walking, suggesting 10,000 steps per day as the minimum amount of walking that should be incorporated into every- day life [2]. Walking refers to the action of transporting the body to another location by repetitive movements of both legs, and is one of the most natural and fundamental actions in humans [3]. It can also be described as a continuous and repetitive movement of individual segments of the body in order to gradually transport the body in a consistent direction, while maintaining the required speed [4]. Health conditions and age can be recognized by a person's gait, relationship between walking speed and walk [5], shoes type and walking [6], and walking on the steps and slope and obstacles [7] etc., there have been many studies to date quantifying and specifying gait.

Received: 16 November, 2015 Revised: 2 December, 2015 Accepted: 2 December, 2015 
Although research on the association between walking and disease has been conducted for many years, recently, there have also been studies using various methods, such as motion analysis, electromyography (EMG) analysis, balance test, to examine the exercise effects of walking, which is the most active activity of daily living.

Various elements are involved in the energy consumption from walking, such as the walker's body weight and walking method [8] and the state of the ground [9]. Upright walking produces forces of impact due to interactions between the feet and the ground, and when excessive movements arise from the continual accumulation of these forces, the joints and muscles can become overloaded, leading to injury [10]. Humans frequently wear shoes to mitigate this impact, and there have been multifaceted studies of alterations to shoes to increase the efficiency of walking exercise [11,12], such as changing the thickness of the soles or the weight of the shoes $[13,14]$.

Shoes are the most basic tool in walking, and help provide stability to prevent injuries to the feet. Hence, the majority of studies on the topic have aimed to modify footwear in a way that improves stability to prevent ankle sprains. However, recently, a new method of shoe design has been conceived that increases the exercise effects rather than function, which induces greater energy consumption and promotes over-exercise; in turn, this provides stability when walking $[15,16]$. Further, there has been persistent development of methods of weight-loading using shoes or sandbags, as a way of increasing the effects of classical exercise [17]. Gwak et al. [18] suggested that the weight-loading method was effective for improving gait efficiency and respiratory circulation, and that increasing weight using shoes was a good method, because it was not limited in terms of the time and place of exercise. However, indiscriminate weight-loading can have a negative effect on the joints of the lower limb, such as arthritis and ligament damage. In fact, Chung [19] detected a weight-dependent change in joint moment, which affected the joint rate of work and the muscle performance, with excessive loads appearing to have a negative effect on joint moment. Recently, light-weight footwear has been emphasized, and while various studies have been conducted on running efficiency and joint moment in relation to lower limb weight-loading, the most effective weight for loading has not been established.

Therefore, the present study aimed to determine a more effective weight for gait training by using weights of $300 \mathrm{~g}$, $500 \mathrm{~g}$, and 1,000 g, with $300 \mathrm{~g}$ being the standard weight for a shoe, and by analyzing the effects of these loads on different lower limb muscles using EMG.

\section{Methods}

\section{Subjects}

Among the subjects who agreed to participate in the experiment after hearing an explanation of the aims and methods, 15 male and 15 female healthy, adult subjects in their 20s who wore shoes for at least 5 hours, 3 times a week, had normal gait and foot shape, and had no internal or external abnormalities in the last year were selected for the study. The physical characteristics of the subjects are summarized in Table 1.

\section{Experimental design}

This study is a non-experimental study that examined the effect of weight of shoes on the muscle fatigue of the lower limbs, and was based on cross-sectional study that examines the state of subjects by single measurement at a certain point.

Based on the method proposed by Gwak et al. [18], the weight of shoes and attachment method was decided within the range between $1 \%$ and $5 \%$, according to the finding that the minimum additional weight is $2 \%$ of the body weight. However, due to circumstances, it was impossible to adjust shoe weight to the body weight of subjects and, therefore, two types of weights were made by using lead, which were then hung on the area close to the ankle, in order to evenly distribute the weight on the anterior-posterior-medial-lateral of

Table 1. Demographic and anthropological characteristics of the subjects

\begin{tabular}{lrrrr}
\hline Characteristic & $300 \mathrm{~g}(\mathrm{n}=10)$ & $500 \mathrm{~g}(\mathrm{n}=10)$ & $1,000 \mathrm{~g}(\mathrm{n}=10)$ & $\mathrm{F}(p)$ \\
\hline Age $(\mathrm{yr})$ & $24.18(2.31)$ & $23.44(0.96)$ & $24.71(1.81)$ & $0.188(0.884)$ \\
Height $(\mathrm{cm})$ & $167.63(2.71)$ & $166.84(3.11)$ & $166.36(2.51)$ & $1.03(0.561)$ \\
Weight $(\mathrm{kg})$ & $64.61(9.43)$ & $62.4(9.70)$ & $65.11(4.43)$ & $0.793(0.322)$ \\
Shoes size $(\mathrm{mm})$ & $247.2(0.81)$ & $245.5(0.47)$ & $246.1(0.42)$ & $0.331(0.721)$ \\
\hline
\end{tabular}

Values are presented as mean (SD). 


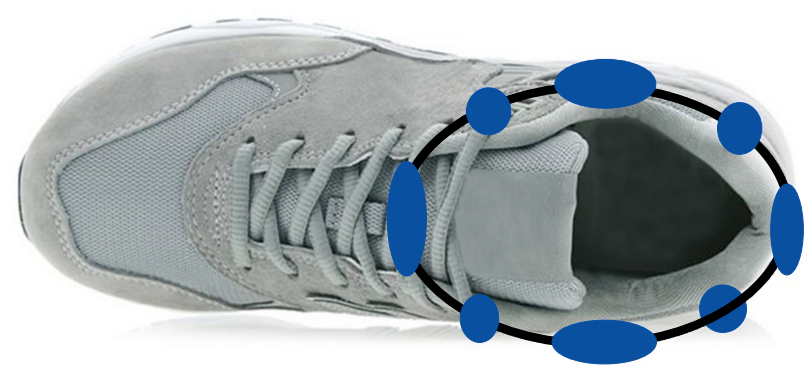

Figure 1. Attachment sites of weight.

the shoes. In the test, the weight of shoes was $300 \mathrm{~g}$ for group $\mathrm{A}, 500 \mathrm{~g}$ for group $\mathrm{B}$, and $1,000 \mathrm{~g}$ for group $\mathrm{C}$, with $300 \mathrm{~g}$ being the basic weight of the shoes, $500 \mathrm{~g}$ being the shoe weight added by $200 \mathrm{~g}$ weight and the weight of light hiking shoes, and 1,000 $\mathrm{g}$ being the shoe weight added by $700 \mathrm{~g}$ weight and the weight of medium-weight hiking shoes.

The weight difference between shoes of different sizes was not taken into account because there is only about $5 \mathrm{~g}$ of difference per $5 \mathrm{~mm}$ of the shoe size.

\section{Procedure}

This experiment used three types of shoe weights $-300 \mathrm{~g}$, $500 \mathrm{~g}$, and $1,000 \mathrm{~g}-$ and to reduce errors in the weight-dependent data, used the same model of shoes from the same brand MRT580DS (Newbalance, Boston, MA, USA) (Figure 1). Measurements were made in the morning to minimize errors due to the accumulation of muscle fatigue during the day, and a prior investigation was performed to identify the appropriate shoe sizes. For the walking exercise, the participants were instructed to arrive at the laboratory 20 minutes before the experiment and were made to sit in a chair and rest for 10 minutes after arrival. The subjects wore artificially weighted shoes and walked on a treadmill in an upright posture for 20 minutes, without rest, at a constant speed of 3.6 $\mathrm{km} / \mathrm{h}$, pertaining to an average walking speed. EMG measurements were taken for 30 seconds during the exercise initiation, and again for 30 seconds at the last 30 seconds prior to the end of exercise, and these measurements were used to calculate the muscle activity and fatigue using the median power frequency (MPF) value with a base value calculated as a percentage during the first and last 30 seconds of walking $[20,21]$.

\section{Measurements}

Muscle fatigue during treadmill walking were measured for the bilateral tibialis anterior, soleus, and medial gastro-

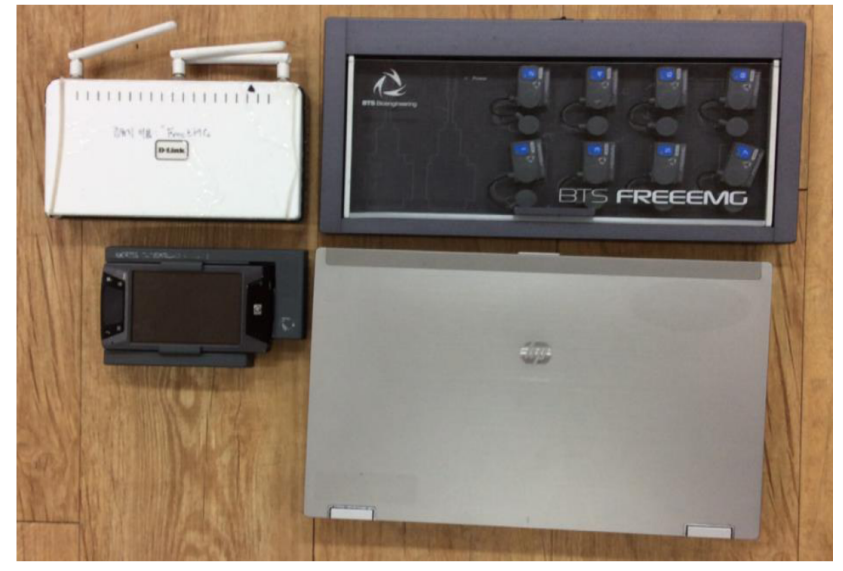

Figure 2. Electromyographic Device. The BTS FREEEMG 300 (BTS Bioengineering, Milan, Italy).

cnemius using BTS FREEEMG 300 (BTS Bioengineering, Milan, Italy) (Figure 2), by a protocol set using Myolab software (BTS Bioengineering). After the measurements, the data were sent to a personal computer using a wireless network device and the participant's protocol was set using Myolab software. In order to avoid technical errors that may affect the results, body hair was removed prior to the experiment, before wiping the area clean with an ethanol swab and attaching the surface electrodes. Disposable 2225H (3M Korea Ltd., Seoul, Korea) electrodes, which are compatible with the BTS FREEEMG 300, were used, and these were placed such that the distance between each electrode EMG probe was attached to the adhesion interval. The electrodes were attached to the locations suggested by the surface electromyography for the non-invasive assessment of muscles [22], as shown in Figure 3. Muscle fatigue was measured based on median frequency of EMG that was generated during the 20 minutes of walking, and muscle activity was based on root mean square, to analyze the eletromyogram patterns.

\section{Data analysis}

Statistical processing in this study was performed using PASW ver. 18.0 for Windows (IBM Co., Armonk, NY, USA). One-way ANOVA (analysis of variance) was used to compare the effects of different loads on muscle fatigue for the three muscles.

Where the one-way ANOVA showed a significant difference, post-hoc testing was performed using Scheffe's test. The statistical significance level was set as $\alpha=0.05$. 


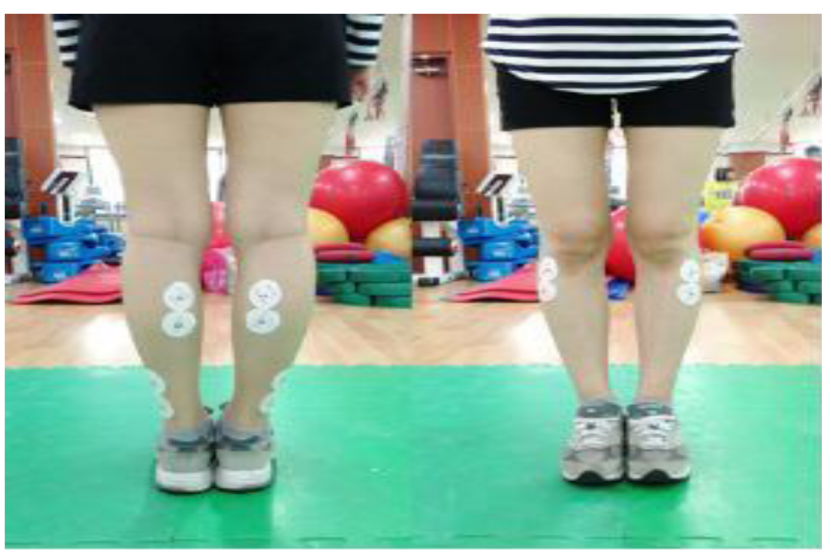

Figure 3. Locations of the surface electromyography electrodes.

\section{Results}

\section{Differences in lower limb muscle fatigue according to shoe weight}

The effects of different shoe weights on muscle fatigue in the lower limbs are shown in Figure 4. Comparison of the mean muscle fatigue according to shoe weight showed that the mean fatigue increased with increasing weight, from 300 $\mathrm{g}$ to $500 \mathrm{~g}$, to $1,000 \mathrm{~g}$. After post-hoc testing, both the right and left soleus muscles showed no significant difference in muscle fatigue between $300 \mathrm{~g}$ and $500 \mathrm{~g}(p>0.05)$, whereas $1,000 \mathrm{~g}$ showed significantly lower median power frequency than $300 \mathrm{~g}$ and $500 \mathrm{~g}(p<0.05)$. Moreover, after post-hoc testing, the right medial gastrocnemius muscle showed a significant difference between $300 \mathrm{~g}$ and $1,000 \mathrm{~g}$ $(p<0.05)$, and the left medial gastrocnemius muscle showed a significant difference between $300 \mathrm{~g}$ and $1,000 \mathrm{~g}(p<0.05)$. Both the right and left tibialis anterior muscles showed no significant difference in muscle fatigue between $300 \mathrm{~g}$ and $500 \mathrm{~g}$ after post-hoc testing, whereas $1,000 \mathrm{~g}$ showed significantly lower median power frequency than $300 \mathrm{~g}$ and $500 \mathrm{~g}(p<0.05)$.

\section{Discussion}

For rehabilitation and improvement of health, an exercise effect needs to be achieved through diverse physical activities suited to the individual patient. However, this is not easy to implement in reality, and hence, recently, to achieve greater efficiency, various methods that effectively utilize movements in activities of daily living, such as standing, walking, or sitting, have been researched. Of these, walking

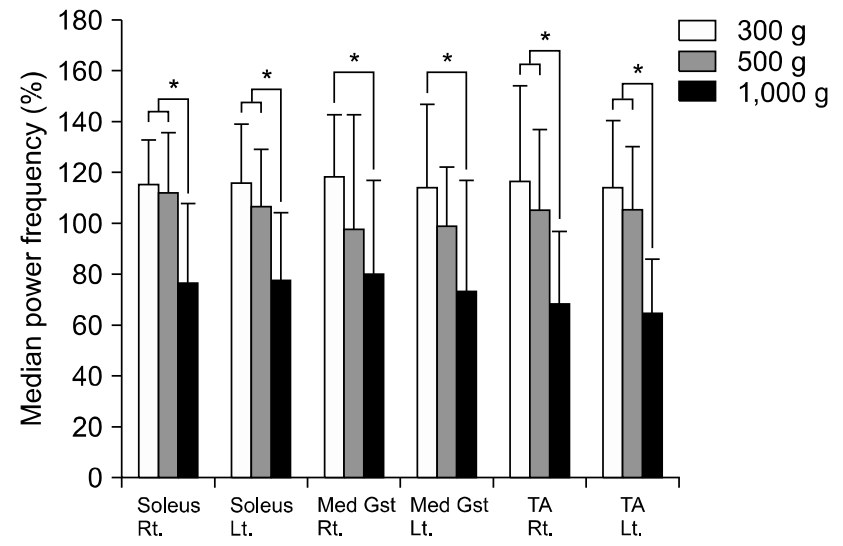

Figure 4. Muscle fatigue analysis. Groups A, B, and C were instructed to wear athletic shoes weighing $300 \mathrm{~g}, 500 \mathrm{~g}$, and 1,000 g, respectively. Rt.: right, Lt.: left, Med: medial, Gst: gastrocnemius, TA: tibialis anterior. ${ }^{*} p<0.05$.

exercise has often been recommended, with multiple studies reporting it to be an effective cardiovascular exercise that does not cause excessive stress to joints and that helps to prevent secondary disease in obese or overweight individuals. Consequently, there has been increasing interest in methods that effectively increase the energy consumption during walking without over-exerting the joints and muscles $[15,23]$.

One way to increase the effects of walking is to alter the hardness, shape, or weight of the shoes, which has been reported to result in changes in energy expenditure and gait patterns when walking. In order to confirm these changes, most studies have investigated changes in body energy consumption and muscle activity after altering the shape or weight of the footwear, and there have been numerous studies reporting an effect on the walking outcomes when shoes are loaded with additional weight $[9,24,25]$.

Hence, the present study aimed to produce a more effective analysis of walking with weighted shoes and to provide more detailed and accurate evidence for the effects thereof. To this end, we used EMG to investigate differences in the fatigue and activity of the lower leg muscles according to different shoe weights.

Previous research has demonstrated an effect of attaching weights to shoes on the respiratory circulation, effects of attaching different weights at different positions, and changes in walking efficiency due to the response of the respiratory system. In the present study, the volunteers were divided into 3 groups according to shoe weight $(300 \mathrm{~g}, 500 \mathrm{~g}$, and $1,000 \mathrm{~g}$ ), and measurements were taken bilaterally from the 
soleus, medial gastrocnemius, and tibialis anterior. The results showed that there was no significant difference in muscle median power frequency between groups $\mathrm{A}$ and $\mathrm{B}$ for the soleus or tibialis anterior, whereas there was a significant decrease in group $\mathrm{C}$ compared to groups $\mathrm{A}$ and $\mathrm{B}(p<0.05)$. Meanwhile, the medial gastrocnemius only showed a significant decrease between groups $\mathrm{A}$ and $\mathrm{C}(p<0.05)$.

Gwak [17] studied the ankle inversion angle, angular speed, ground reaction force, and impact with different shoe weights, although this study used weight categories of $150 \mathrm{~g}$, $250 \mathrm{~g}$, and $350 \mathrm{~g}$. The results showed a decrease in ground reaction force is that $150 \mathrm{~g}$ is $1,902 \mathrm{~N}, 250 \mathrm{~g}$ is $1,855 \mathrm{~N}, 350$ $\mathrm{g}$ is $1,822 \mathrm{~N}$ as the weight increased to $350 \mathrm{~g}$, in addition to a decrease in the peak impact force 1,407 N, 1,403 N, 1,302 $\mathrm{N}$ with increasing shoe weight. Combined with the results of our study, as the weight of footwear increases, in accordance with the equation $\mathrm{F}=\mathrm{M} \cdot \mathrm{A}$, the force that the body imparts on the ground also increases, and by the law of action-reaction, this seems to result in greater absorption of the reactionary force and impact force between the shoes and ground. In addition, a study by Boozari et al. [26] comparing normal and flat feet found that muscle fatigue was higher for flat feet, and that during walking, the arch collapsed in flat feet, meaning that it was unable to absorb the impact between the body and the ground, thus leading to fatigue. Hence, a significant association can be predicted between impact force and muscle fatigue, and this suggests that reductions in the ground reaction force and impact force causes a reduction not only in the impact conveyed to the body, but also in muscle fatigue. Furthermore, in one previous study [17], the ankle inversion angle did not change significantly with increasing shoe weight, but rather increased somewhat. Biomechanically, an increase in the ankle inversion angle would increase the activity in the medial gastrocnemius during walking.

Although the present study did not find a significant difference in medial gastrocnemius fatigue between groups $\mathrm{A}$ and $\mathrm{B}$ or groups $\mathrm{B}$ and $\mathrm{C}$, there was a significant difference between groups $\mathrm{A}$ and $\mathrm{C}$, which had a weight difference of $700 \mathrm{~g}$. This also appears to be relevant when compared with the previous study showing no significant change in ankle inversion angle with increase weight, and with more thorough research into the effects on the ankle inversion angle with different shoe weights, it might be possible to achieve even more effective rehabilitation or exercise by utilizing different shoes.

With reference to previous studies on shoe weight, Gwak et al. [18] found a significant increase in the heat rate of male is $115,117,121,123,126$, female is $118,122,135,141,144$, 146 ( $p>0.05$ ) with increased shoe weight, in addition to significant increases in oxygen consumption, carbon dioxide emission, and energy consumption. Further, according to a study by Kim [27], who studied changes in respiratory function and energy consumption according to shoe weight and walking speed, while there was no significant difference in energy consumption and respiratory function with different shoe weights at normal walking speed, at a fast walking speed, there was a significant difference. These previous studies suggest that the respiratory function and energy metabolism were stimulated by increased load during walking, and as a reason for this, changes in energy consumption due to increased muscle activity required for walking under heavier loads cannot be excluded.

Comparing these results with those of the present study, the overall muscle activity increased, but only the tibialis anterior showed a significant increase, while the gastrocnemius and soleus showed non-significant increases. The joint for the gastrocnemius and soleus is a class 2 lever, and due to its mechanical properties, even a small force produces a large force in response. Meanwhile, the tibialis anterior is a class 3 lever, and it is thought to show more activity during walking because it has minimal mechanical gain. Given that the tibialis anterior is a muscle that always needs to be attended to in ankle sprain patients, it seems that it may be possible to effectively exercise the tibialis anterior and reduce muscle fatigue by using weighted shoes.

Furthermore, the study by Kim [27] showed a significant weight-dependent difference in energy consumption for rapid walking 380 to $511 \mathrm{cal}$ but 373 to $456 \mathrm{cal}$ not for normal walking, demonstrating that more significant differences can be seen when there is more work. Similarly, in the present study, it was considered that a significant difference might have been seen if the experiment was conducted for a longer time, or at a faster walking pace.

In conclusion, this study provided a scale for the choice of footwear by quantifying the effects of different shoe weights on muscle fatigue in the lower limbs. The results of the present study can be used as basic data for rehabilitation or exercise using walking, and suggest that rather than light and comfortable footwear, selecting footwear suited to the symptoms, requirements, and conditions of the individual, such as the active use of weight-loading, can be helpful in achieving the therapist's goals in an improved environment, by effective rehabilitation and reduction of fatigue. When 
performing physiotherapy for patients with difficulty walking, it is thought that heavier shoes may be able to contribute to reducing muscle fatigue and correcting gait patterns.

This study had limitations because, first, the maximum available number of probes of BTS FREEEMG was eight, and, therefore, the study did not measure various muscles while walking. Second, due to difficulties with making shoes, weights were added to shoes instead of using shoes that were suitable for the weight. The result of this study can be used for future research and shoe manufacturers. Lastly, only a small number of subjects was used considering the design of the test, because only subjects who satisfied the relevant criteria were selected.

\section{Conflict of Interest}

The authors declared no potential conflicts of interest with respect to the authorship and/or publication of this article.

\section{References}

1. Popkin BM, Adair LS, Ng SW. Global nutrition transition and the pandemic of obesity in developing countries. Nutr Rev 2012;70:3-21.

2. Tudor-Locke C, Craig CL, Brown WJ, Clemes SA, De Cocker K, Giles-Corti B, et al. How many steps/day are enough? For adults. Int J Behav Nutr Phys Act 2011;8:79.

3. Chao EY, Rim K. Application of optimization principles in determining the applied moments in human leg joints during gait. $\mathrm{J}$ Biomech 1973;6:497-510.

4. Perry J. Gait analysis: normal and pathological function. Thorofare, NJ: Slack; 1992. p. 413-22.

5. Helbostad JL, Moe-Nilssen R. The effect of gait speed on lateral balance control during walking in healthy elderly. Gait Posture 2003;18:27-36.

6. Ramstrand N, Thuesen AH, Nielsen DB, Rusaw D. Effects of an unstable shoe construction on balance in women aged over 50 years. Clin Biomech (Bristol, Avon) 2010;25:455-60.

7. Son H, Kim K. A kinematic analysis of patients with knee osteoarthritis during gait on level ground, ramps and stairs. J Phys Ther Sci 2013;25:277-80.

8. VanSwearingen JM, Perera S, Brach JS, Cham R, Rosano C, Studenski SA. A randomized trial of two forms of therapeutic activity to improve walking: effect on the energy cost of walking. J Gerontol A Biol Sci Med Sci 2009;64:1190-8.

9. Bosco C, Saggini R, Viru A. The influence of different floor stiffness on mechanical efficiency of leg extensor muscle. Ergonomics 1997;40:670-9.
10. Kim TW, Kim JT. Analysis of muscle powers according to different walking speeds in obese and normal people. Korean J Sport Sci 2007;18:497-510.

11. Fong DT, Hong Y, Li JX. Cushioning and lateral stability functions of cloth sport shoes. Sports Biomech 2007;6:407-17.

12. Wezenberg D, de Haan A, van Bennekom CA, Houdijk H. Mind your step: metabolic energy cost while walking an enforced gait pattern. Gait Posture. 2011;33:544-9.

13. Sekizawa K, Sandrey MA, Ingersoll CD, Cordova ML. Effects of shoe sole thickness on joint position sense. Gait Posture. 2001; 13:221-8.

14. Murley GS, Landorf KB, Menz HB. Do foot orthoses change lower limb muscle activity in flat-arched feet towards a pattern observed in normal-arched feet? Clin Biomech (Bristol, Avon) 2010;25:728-36.

15. Nigg B, Hintzen S, Ferber R. Effect of an unstable shoe construction on lower extremity gait characteristics. Clin Biomech (Bristol, Avon) 2006;21:82-8.

16. Romkes J, Rudmann C, Brunner R. Changes in gait and EMG when walking with the Masai Barefoot Technique. Clin Biomech (Bristol, Avon) 2006;21:75-81.

17. Gwak CS. Effects of shoe weight and midsole hardness of running shoes on running economy and its application. Korean $\mathbf{J}$ Phys Educ 2001;40:955-73.

18. Gwak CS, Lee GS, Kim HS. Effects of shoe weights on the walking economy. Korean J Phys Educ 2003;42:677-86.

19. Chung HK. The changes of joint moments according to weight loading gait on normal adults. J Korean Acad Phys Ther 2003; 10:53-61.

20. Cheung JT, Zhang M, An KN. Effects of plantar fascia stiffness on the biomechanical responses of the ankle-foot complex. Clin Biomech (Bristol, Avon) 2004;19:839-46.

21. Hong Y, Li JX, Fong DT. Effect of prolonged walking with backpack loads on trunk muscle activity and fatigue in children. $\mathrm{J}$ Electromyogr Kinesiol 2008;18:990-6.

22. Hermens HJ, Freriks B, Disselhorst-Klug C, Rau G. Development of recommendations for SEMG sensors and sensor placement procedures. J Electromyogr Kinesiol 2000;10:361-74.

23. Seo YW, Lee HJ, Yun KE, Park HS. Energy intake and resting energy expenditure of middle-aged obese korean women. Korean J Obes 2009;18:31-7.

24. Long JT, Klein JP, Sirota NM, Wertsch JJ, Janisse D, Harris GF. Biomechanics of the double rocker sole shoe: gait kinematics and kinetics. J Biomech 2007;40:2882-90.

25. Vanderpool MT, Collins SH, Kuo AD. Ankle fixation need not increase the energetic cost of human walking. Gait Posture 2008; 28:427-33

26. Boozari S, Jamshidi AA, Sanjari MA, Jafari H. Effect of functional fatigue on vertical ground-reaction force in individuals with flat feet. J Sport Rehabil 2013;22:177-83.

27. Kim UG. The effects of cardiorespiratory response and energy economy to walking exercise with added shoe weight, and to verify the shoe weight and walking economy. Korea Sport Res 2005; $16: 155-64$ 